\title{
Differentiation between systolic and diastolic dysfunction
}

\author{
M. Federmann and O. M. Hess \\ Cardiology, Department of Internal Medicine, University Hospital of Zürich, Switzerland
}

KEY WORDS: Systolic dysfunction, diastolic dysfunction, left ventricular relaxation, left ventricular filling, passiveelastic properties, mitral filling patterns, medical treatment.

Lefi ventricular ( $L V$ ) failure can be divided into systolic and diastolic dysfunction. The former is characterized by a reduced ejection fraction and an enlarged $L V$ chamber, the latter by an increased resistance to filling with increased filling pressures. Systolic dysfunction is clinically associated with left ventricular failure in the presence of marked cardiomegaly, while diastolic dysfunction is accompanied by pulmonary congestion together with a normal or only slightly enlarged ventricle. Echocardiography is currently the most relevant technique for non-invasive differentiation of the two forms.

Systolic dysfunction is easily assessable by estimation of global ejection fraction and regional wall motion. Diastolic dysfunction can be diagnosed indirectly by means of a normal or nearly normal ejection fraction and changes of the mitral filling pattern in the context of $L V$ failure. For an exact determination of dibstolic dysfunction $L V$ catheterization is required Systolic dysfunction treatment is well defined, consisting of $A C E$ inhibitors, followed by diuretics and digitalis. Calcium channel blockers are usually contraindicated. Diastolic dysfunction therapy is more dependent on the underlying disease Calcium channel blockers, ACE inhibitors or beta-blockers are first line drugs in most instances: diuretics can be added with increasing symptoms. Digitalis should be avoided, except in atrial fibrillation, to control heart rate.

\section{Introduction}

Chronic heart failure (CHF) is generally associated with a poor prognosis, with the annual mortality rate ranging between 20 and $50 \%$ depending on the severity of cardiac dysfunction ${ }^{[1]}$. Heart failure has been subdivided into different forms such as forward and backward failure, highand low-output failure, systolic or diastolic dysfunction etc. All these entities show considerable variations in regard to clinical course and prognosis. During the last two decades, the role of diastolic dysfunction in the development of heart failure has been increasingly recognized. The different pathophysiology of systolic and diastolic dysfunction affects prognosis and therapeutic management.

\section{Definition and aetiology}

Systolic dysfunction is determined by an impaired pump function with reduced ejection fraction and an enlarged end-diastolic chamber volume ${ }^{[2]}$. Diastolic dysfunction is characterized by an increased resistance to filling with increased filling pressures ${ }^{[2]}$. Isolated systolic dysfunction is rare; most patients with CHF have predominating systolic dysfunction with some degree of diastolic impairment. The main aetiology of systolic dysfunction is coronary artery disease followed by hypertensive heart disease ${ }^{[3.4]}$. Other, but less frequent causes are dilated cardiomyopathy and myocarditis. Left ventricular (LV) failure is caused by isolated diastolic dysfunction in up to $40 \%$ of all cases ${ }^{[5]}$, mainly due to long-standing hypertension ${ }^{[3,4\}}$. Coronary artery disease is secondary; hypertrophic cardiomyopathy, restrictive cardiomyopathies and aortic valve

Correspondence. Prof Dr O. M. Hess, Cardiology, Department of Internal Medicine, University Hospital, 8091 Zurich, Switzerland. diseases are less frequent. Age also has a bearing on physiological diastolic dysfunction ${ }^{[6}$.

\section{Pathophysiology}

The pathophysiology of systolic dysfunction begins with a reduction in systolic pump function following a loss of muscle cells, a decrease in myocardial contractility, and/or structural changes of the myocardium with an increase in interstitial fibrosis (= myocardial remodelling). The consecutive fall in cardiac output is counteracted by two major compensatory mechanisms, the Frank-Starling mechanism and activation of the sympathetic nervous system ${ }^{[7]}$. The Frank-Starling mechanism leads to an enlargement of the

Table 1 The three major diastolic functional abnormalities and their main aetiology

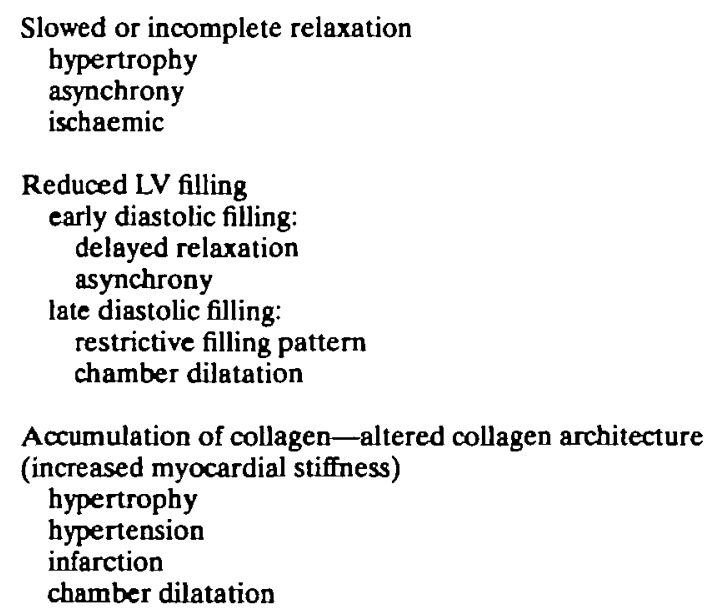

Accumulation of collagen-altered collagen architecture (increased myocardial stiffness)

hypertrophy

hypertension

infarction

chamber dilatation 


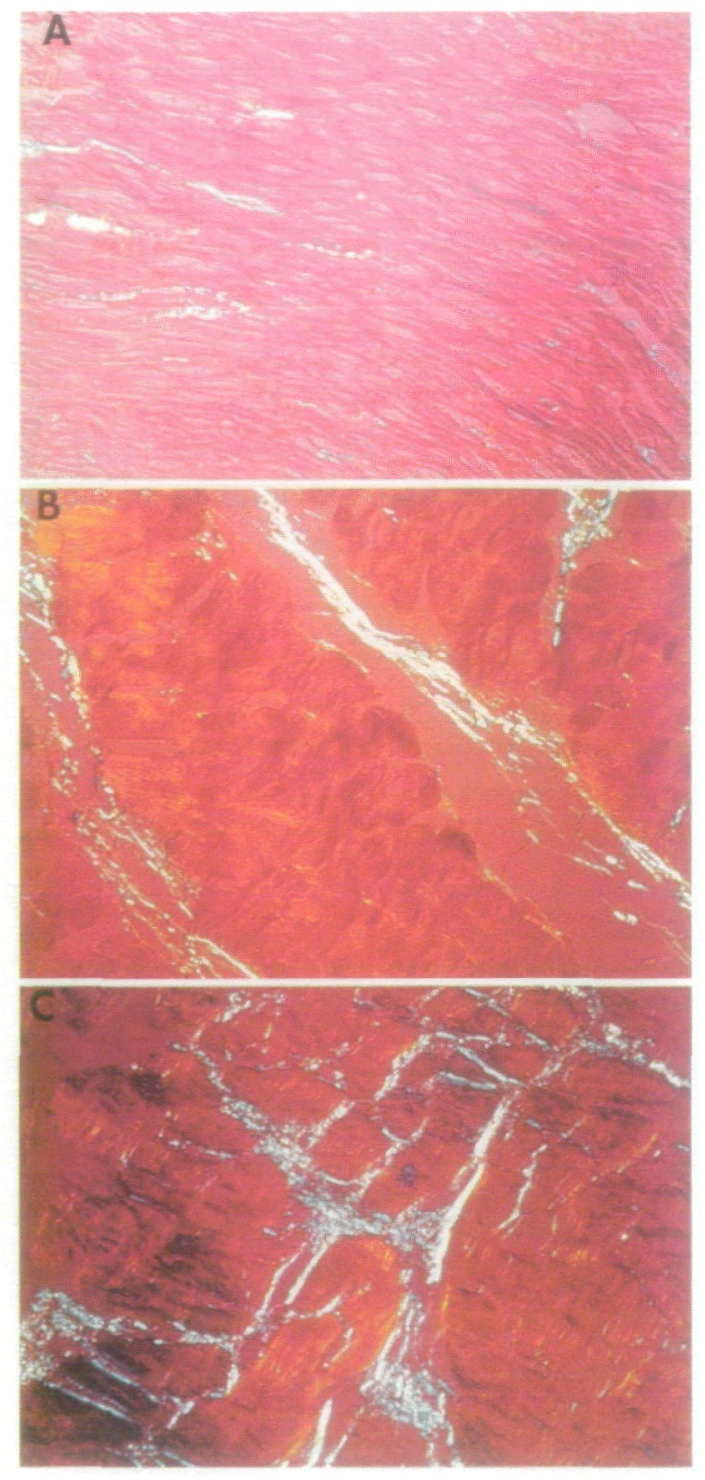

Figure 1 Photomicrographs (magnification $\times 300$ and reproduced here at $80 \%$ ) of different patterns of collagen accumulation and altered collagen architecture. Differently orientated collagen fibres are represented by white or blue in the polarized light microscope. (A) Normal myocardium with few, tiny collagen fibres. (B) Biopsy sample from a patient with aortic valve disease showing an increase in interstitial collagen fibres, which are mostly orientated longitudinally to muscle fibres. (C) Biopsy sample from another patient with aortic valve disease. The collagen concentration is increased with evidence of extensive malalignment of collagen fibres (= cross-hatching).

chamber and an increase in end-diastolic pressure. Sympathetic activation results in an increase in the heart's inotropic and chronotropic state. The adverse effects of these compensatory mechanisms-higher wall stress and higher oxygen requirements-are reduced by subsequent myocardial hypertrophy, activation of the baroreceptor reflex and release of atrial natriuretic peptide. The baroreceptor reflex inhibits sympathetic activation and atrial natriuretic peptide exerts diuretic and vasodilating effects.
During the development of chronic heart failure, these primary and secondary compensatory mechanisms lose their beneficial influences, for instance by a down-regulation of the beta-receptors and dysfunction of the baroreceptor reflex. A vicious circle evolves, which is enhanced by increasing activation of the renin-angiotensin system and by the release of vasopressin, with subsequent vasoconstriction and water retention.

The pathophysiology of diastolic dysfunction is generally more diverse, depending on the underlying disease. Factors extrinsic to the ventricular chamber, such as pericardial constriction or ventricular interdependence must be distinguished from those intrinsic to the chamber ${ }^{[8]}$. There are three major diastolic functional abnormalities, namely slowed or incomplete relaxation, reduced LV filling, and altered passive elastic properties, especially due to accumulation of collagen or altered collagen architecture (Table 1) ${ }^{[9]}$. On the cellular level, an increase in myocyte calcium content, a decrease in ATP levels and an activation of angiotensin II seem to be responsible for impaired relaxation ${ }^{[10]}$. Alterations of the passive elastic properties are probably the result of enhanced interstitial fibrosis and changes in the collagen structure. A change in the collagen architecture with an abnormal alignment (cross-hatching) and/or endomyocardial fibrosis appears to be responsible for an increase in myocardial stiffness, whereas the sole increase in collagen leaves passive diastolic function unaltered (Figs 1 and 2$)^{[1]}$.

LV diastolic dysfunction results in an elevation of ventricular and atrial filling pressures, with consecutive pulmonary congestion. In this situation, atrial contraction becomes more important ${ }^{[2]}$. Chronic atrial enlargement favours the onset of atrial fibrillation. This is poorly tolerated in patients with diastolic dysfunction and results often in acute $\mathrm{CHF}^{[8]}$. An accompanying tachycardia contributes

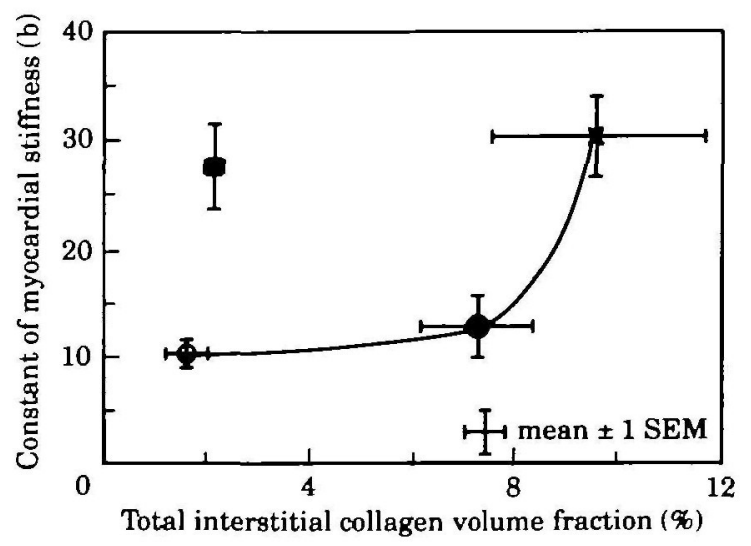

Figure 2 Plot of the constant of myocardial stiffness (b) vs the total collagen volume fraction in normals and patients with aortic valve disease and different amounts of myocardial collagen and/or architecture. The relationship is exponential and increases only slightly from normal $(O)$ to enhanced myocardial collagen volume fraction (-), but increases significantly in the presence of crosshatching and/or endomyocardial fibrosis $(x)$. Likewise, isolated cross-hatching and/or endomyocardial fibrosis ( $\boldsymbol{\square}$ left upper point) is associated with a significant increase of myocardial stiffness Reproduced with permission from the American College of Cardiology ${ }^{[11]}$. 


\section{phase I: $\quad$ phase II:}

Systolic compensation

Systolic compensation with diastolic failure phase III:

Systolic and diastolic failure
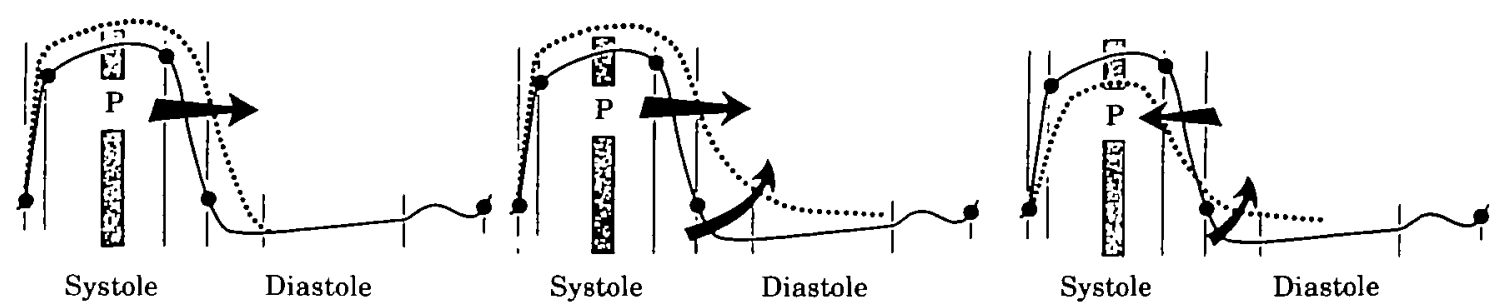

Figure 3 Pressure (P)-time relationship of the left ventricle in successive phases during the pathophysiological evolution of pressure or volume overloading, or both (modified from Brutsaert et al ${ }^{[13]}$, reproduced with permission). There are three different phases. (1) Systolic compensation: prolonged contraction leads to retarded (but not prolonged) relaxation with shortening of diastole. (2) Systolic compensation with diastolic failure; impaired relaxation and increased myocardial stiffness occur resulting in elevated left ventricular diastolic pressures. (3) Systolic and diastolic failure: in a third phase, systolic failure develops.

to diastolic failure by shortening of diastolic filling time. During the clinical course, chronic mechanical overload and subendocardial hypoperfusion induce further hypertrophy and interstitial fibrosis (= myocardial remodelling) and finally systolic dysfunction may occur. According to Brutsaert and co-workers ${ }^{[13]}$ three phases can be distinguished in the evolution of diastolic dysfunction in patients with chronic pressure and volume overload (Fig. 3). Initially a prolongation of systolic contraction is associated with retarded relaxation, which shortens diastolic filling time. In a second phase, impairment of myocardial relaxation occurs, which (along with increased stiffness and elevated heart rate) may result in enhanced diastolic filling pressures. In a third phase, concomitant systolic dysfunction develops.

\section{Clinical symptoms and diagnostic approach}

In clinical practice, systolic dysfunction is characterized by the occurrence of CHF in the presence of marked cardiomegaly. Diastolic dysfunction is associated with pulmonary congestion and a normal or only slightly enlarged ventricle.

Echocardiography is currently the most relevant noninvasive method for differentiation of systolic and diastolic dysfunction. Systolic performance of the ventricle can be quantified by regional and global function parameters such as LV-ejection fraction ${ }^{[14]}$ and regional wall motion analysis. In contrast, diastolic function cannot be estimated directly because the measurable filling parameters are a function of multiple determinants. Diastolic dysfunction is often associated with typical, but non-specific, mitral filling patterns (Fig. 4), such as the pattern of delayed relaxation with increased isovolumic relaxation time and a low E/A ratio, and the restrictive pattern with shortened isovolumic relaxation time and a high $\mathrm{E} / \mathrm{A}$ ratio ${ }^{[15]}$.

During the course of progressive diastolic dysfunction, mitral filling may shift from the first to the second pattern with a 'pseudonormalization' in between. Additional information for interpretation of mitral filling pressures may be gained from the pulmonary venous flow velocity pattern and the colour Doppler M-mode display of mitral inflow ${ }^{[15]}$. Radionuclide angiography, another non-invasive diagnostic method, has similar advantages and limitations in the assessment of diastolic dysfunction.

Only LV catheterization allows reliable assessment of LV diastolic function and differentiates between abnormal
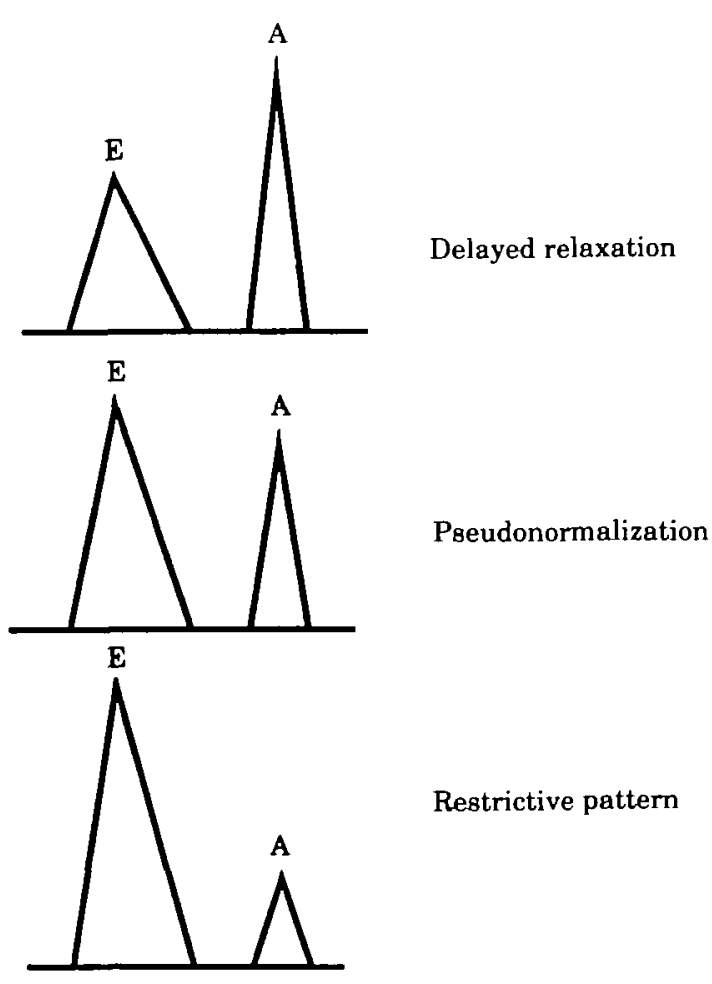

Figure 4 Typical, but non-specific mitral filling patterns in diastolic dysfunction. The pattern of delayed relaxation is characterized by a low amplitude of the E-wave due to a reduced early left ventricular filling: $\mathrm{E} / \mathrm{A}$-ratio $<1$. In the course of diastolic dysfunction, a 'pseudonormalization' with a 'normal' E/A-ratio ( $>1)$ can occur. In advanced forms of diastolic dysfunction a restrictive pattern with a high $\mathrm{E}$-wave, a small $\mathrm{A}$-wave and an increased $\mathrm{E} / \mathrm{A}$ ratio $(>2)$ can be observed. 


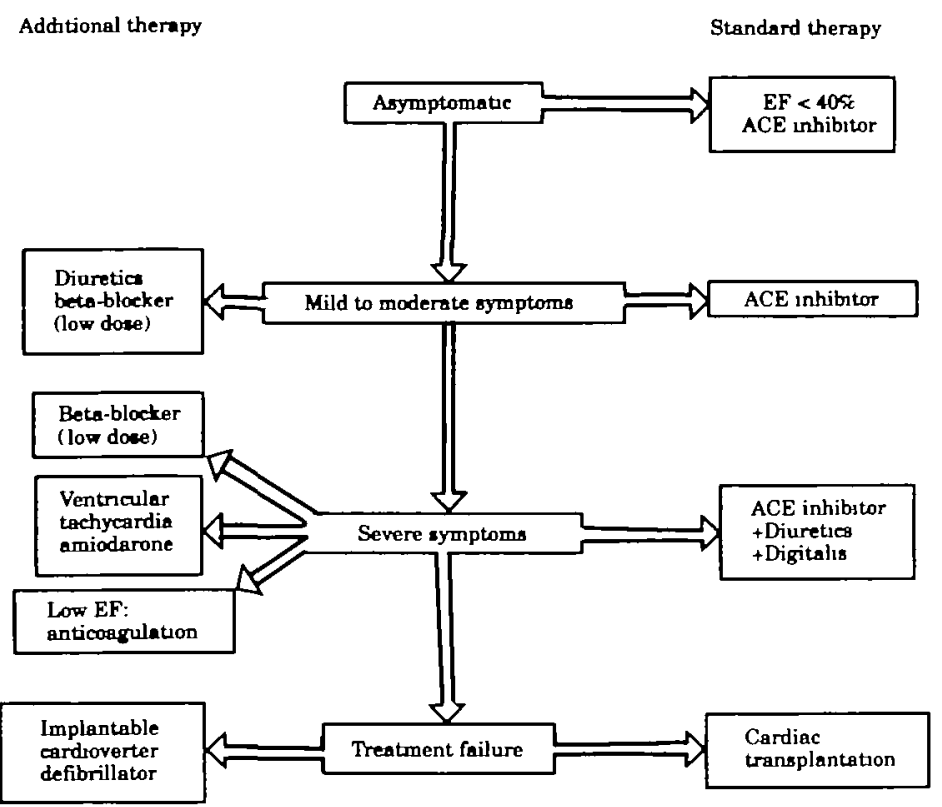

Figu:e 5 Therapeutic strategies based on clinical symptoms in patients with systolic dysfunction.

relaxation and altered passive elastic properties. LV relaxation can be quantified from the rate of LV pressure decay, and LV stiffness from the simultaneous pressure-volume (chamber stiffness) or stress-strain (myocardial stiffness) relationship. For clinical purposes this differentiation is rarely necessary, but might help to define the severity of diastolic dysfunction in individual patients.

\section{Prognosis}

The prognosis of systolic dysfunction is poor, with a 5year survival rate close to $40 \%$ in most studies, compared to $70 \%$ in patients with isolated diastolic dysfunction ${ }^{[4]}$. Diastolic dysfunction often precedes systolic dysfunction and represents an early form of heart failure, not necessarily accompanied by clinical symptoms. However, when sys-

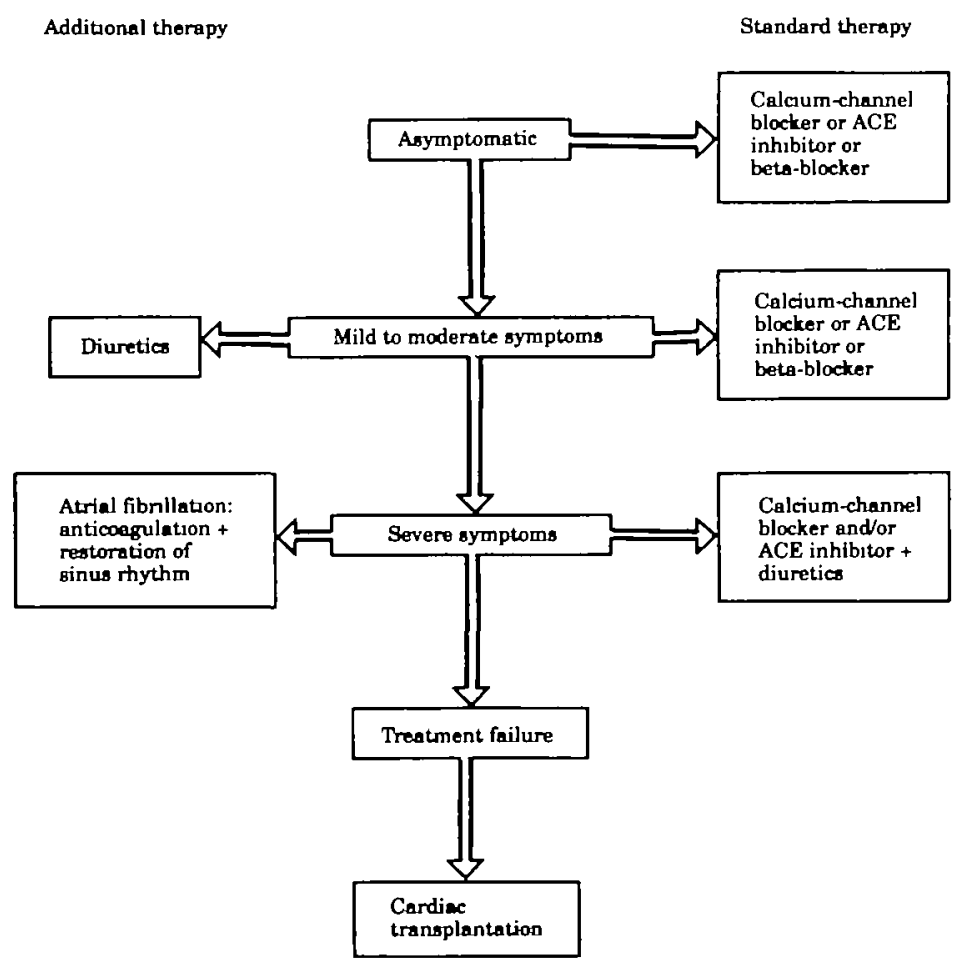

Figure 6 Therapeutic strategies based on clinical symptoms in patients with diastolic dysfunction, such as in hypertensive heart disease. 
tolic dysfunction ensues, the heart failure process is usually in an advanced stage involving both systolic and diastolic function and thus has a poor outcome.

\section{Therapy}

Medical treatment in systolic dysfunction (Fig. 5) is primarily based on ACE inhibitors, drugs that are effective even in asymptomatic patients with an LV ejection fraction of $35 \%{ }^{[16]}$ or $40 \%$ in patients after myocardial infarction ${ }^{[17]}$. With further progression of heart failure, diuretics followed by digitalis are employed ${ }^{[18]}$. Beta-blockers in low doses seem to achieve an additional benefit ${ }^{[19]}$. Calcium channel blockers are rarely indicated because of their negative inotropic effects. Newer calcium antagonists, such as amlodipine, nisoldipine or felodipine might improve symptoms of systolic dysfunction ${ }^{[20]}$, but this has to be proven in large scale trials. Atrial fibrillation and ventricular tachycardia are preferably treated by amiodarone. Anticoagulation to prevent systemic embolism is recommended in severe systolic dysfunction, especially in dilated cardiomyopathy ${ }^{[2]]}$. In treatment failure, cardiac transplantation is possible in appropriate patients; otherwise intractable life-threatening ventricular arrhythmias can be managed by means of an implantable cardioverter defribrillator.

Data are sparse for medical treatment of diastolic dysfunction. The underlying disease is currently the most important determinant for therapy. In hypertensive heart disease for example (Fig. 6), therapy may start with a calcium channel blocker, an ACE inhibitor, or a beta-blocker. All these drugs have been shown to reduce left ventricular hypertrophy ${ }^{[2]}$. A diuretic can be added when symptoms increase, and in advanced stages a combination of these drugs may be necessary. When atrial fibrillation occurs, restoration of sinus rhythm is often mandatory and is best achieved with electric conversion. For prophylaxis of recurrence, beta-blockers, especially sotalol, which exerts a class III antiarrhythmic effect, are suitable. Cardiac transplantation is rarely indicated. The antifibrotic action of spironolactone is currently being investigated: promising animal data show that spironolactone can prevent myocardial fibrosis ${ }^{[2]}$. Digitalis can worsen LV relaxation and should be avoided, except in atrial fibrillation.

\section{References}

[1] Hess OM. Pathophysiologie der Herzinsuffizienz. Schweiz med Wschr 1990, 120: 1833-7.

[2] Hess OM. Hemodynamics in cardiac failure: systolic and diastolic dysfunction. Ther Umsch 1993; 50: 414-8.

[3] Dougherty AH, Naccarelli GV, Gray EL, Hicks $\mathrm{CH}$, Goldstein RA. Congestive heart failure with normal systolic function. Am J Cardiol 1984; 54: 778-82.
[4] Cohn JN, Johnson GJ, Veterans Administration Cooperative Study Group. Heart failure with normal ejection fraction: The V-Heft study. Circulation 1990; 81 (Suppl III): III-48-III-53.

[5] Soufer R, Wohlgelernter D, Vita NA et al. Intact systolic function in clinical congestive heart failure. Am J Cardiol 1985; 55: 1032-6.

[6] Nixon JV, Burns CA. Cardiac effects of aging and diastolic dysfunction in the elderly. In: Gaasch WH, LeWinter MM, eds. Left ventricular diastolic dysfunction and heart failure Philadelphia: Lea and Febiger, 1993: 427-35.

[7] Packer M. Pathophysiology of chronic heart failure. Lancet 1992; 340: 88-92.

[8] Grossman W. Diastolic dysfunction and congestive heart failure. Circulation 1990, 81 (Suppl III): III-1-III-7.

[9] Hess OM. Diastolische Dysfunktion. Schweiz med Wschr 1991; 1942-5.

[10] Apstein CS, Morgan JP. Cellular mechanisms underlying left ventricular diastolic dysfunction. In: Gaasch WH, LeWinter MM, eds. Left ventricular diastolic dysfunction and heart failure. Philadelphia: Lea and Febiger, 1993: 3-24.

[11] Villari B, Campbell SE, Hess OM et al. Influence of collagen network on left ventricular systolic and diastolic function in aortic valve disease. J Am Coll Cardiol 1993; 22: 1477-84.

[12] Little WC, Downes TR. Clinical evaluation of left ventricular diastolic performance. Progr Cardiovasc Dis 1990; 32: 273-90.

[13] Brutsaert DL, Sys SU, Gillebert TH. Diastolic failure: Pathophysiology and therapeutic implications. J Am Coll Cardiol 1993; 22: 318-25.

[14] Jenni R, Vieli A, Hess OM, Anliker M, Krayenbuehl HP. Estimation of left ventricular volume from apical orthogonal 2-D echocardiograms. Eur Heart J 1981; 2: 217-25.

[15] Thomas JD. Doppler echocardiography and left ventricular diastolic dysfunction. In: Gaasch WH, LeWinter MM, eds. Left ventricular diastolic dysfunction and heart failure. Philadelphia: Lea and Febiger, 1993: 192-218.

[16] The SOLVD Investigators. Effect of enalapril on mortality and development of heart failure in asymptomatic patients with reduced left ventricular ejection fraction. $N$ Engl J Med 1992; 327: 685-91.

[17] Pfeffer MA, Braunwald E, Moyé A et al Effect of captopril on mortality and morbidity in patients with left ventricular dysfunction after myocardial infarction. Results of the Survival and Ventricular Enlargement Trial. N Engl J Med 1992; 327: 669-77.

[18] Follath F. Medikamentöse Therapie bei Herzinsuffizienz. Ther Umsch 1993; 50: 425-33.

[19] Hjalmarson A, Waagstein F. New therapeutic strategies in chronic heart failure: challenge of long-term beta-blockade. Eur Heart J 1991; 12 (Suppl F): 63-9.

[20] Packer M. Treatment of chronic heart failure. Lancet 1992; 340: 92-5.

[21] Fuster V, Stein B, Halperin JL, Chesebro JH. Antithrombotic therapy in cardiac disease: an approach based on pathogenesis and risk stratification. Am J Cardiol 1990; 65: 38C-44C.

[22] Dahlof B, Pennert K, Hansson L. Reversal of left ventricular hypertrophy in hypertensive patients. A metaanalysis of 109 treatment studies Am Heart J 1992; 5: 95-110.

[23] Brilla CG, Matsubara LS, Weber KT. Antifibrotic effects of spironolactone in preventing myocardial fibrosis. Am J Cardiol 1993; 71: 12A-16A. 